\title{
Von Reallaboren zu urbanen Experimenten: deutsche und internationale Debatten
}

\author{
Kristine Kern (1), Wolfgang Haupt @ \\ Eingegangen: 1. August 2020 - Angenommen: 23. Juli 2021 - Online veröffentlicht: 9. August 2021
}

\section{Zusammenfassung}

In den letzten Jahren sind Reallabore zu einem wichtigen Bestandteil der anwendungsbezogenen Nachhaltigkeitsforschung in Deutschland und Europa geworden. Derzeit verlagert sich die internationale wissenschaftliche Debatte mehr und mehr auf die Untersuchung von lokalen Experimenten. Vor diesem Hintergrund dient dieser Beitrag vor allem dem Zweck, die deutschsprachige Forschung zu Reallaboren innerhalb der europäischen und internationalen Debatten zu verorten und systematisch mit den Debatten um Urban Living Labs und Experimenten zu vergleichen. In diesem Zusammenhang konzentrieren wir uns vor allem auf die Grundannahmen dieser drei Forschungsansätze, ihre Entstehung und Anwendungsbereiche sowie die Bedeutung von Lernprozessen. Obwohl alle drei Ansätze auf urbane Nachhaltigkeitstransformationen abzielen, ist nur wenig über die mittel- und langfristigen Wirkungen solcher Ansätze bekannt. Deren zeitliche und räumliche Skalierung wurde zwar als Problem erkannt, aber bislang kaum systematisch untersucht. Der Beitrag zielt darauf ab, die deutsche Debatte um Reallabore zu bereichern und sie mit der internationalen Diskussion zu verbinden.

Schlüsselwörter: Reallabore - Urban Living Labs Experimente - Skalierung - Nachhaltigkeitstransformation

Prof. Dr. Kristine Kern, Leibniz-Institut für Raumbezogene Sozialforschung (IRS), Flakenstraße 29-31, 15537 Erkner, Deutschland kristine.kern@leibniz-irs.de

Åbo Akademi University, Fänriksgatan 3B, 20500 Turku, Finnland

Dr. Wolfgang Haupt, Leibniz-Institut für Raumbezogene Sozialforschung (IRS), Flakenstraße 29-31, 15537 Erkner, Deutschland wolfgang.haupt@leibniz-irs.de

(c) (1) (c) 2021 Kern; licensee oekom verlag. This Open Access article is published under a Creative Commons Attribution 4.0 International License.

\section{From Real-World Labs to Urban Experiments: German and International Debates}

\begin{abstract}
In recent years, real-world labs have become an important part of application-related sustainability research in Germany and Europe. Currently, the international academic debate is shifting more and more towards research on local experiments. Against this backdrop, this paper serves in particular the purpose of placing the German-speaking research on realworld labs within international debates, and of comparing it systematically with debates on urban living labs and experiments. In this context, we concentrate especially on the basic assumptions of these three approaches, their origins and areas of application as well as the importance of learning processes. Although all three approaches aim at urban sustainability transformations, not much is known about their medium- and long-term impacts. Their temporal and spatial scaling, which is critical here, was recognized as problem but has barely become object of systematic research. This paper follows the purpose to enrich the German debate on realworld labs and to link it to the international discussion.
\end{abstract}

Keywords: Real-World Labs — Urban Living Labs * Experiments $=$ Scaling $=$ Sustainability transition

\section{Einleitung}

Die Untersuchung von Reallaboren hat international eine lange Tradition, zu einem festen Bestandteil der transdisziplinären Forschung in Deutschland wurde sie jedoch erst in den letzten Jahren. Zwar verbindet man mit dem Begriff Labor vor allem naturwissenschaftliche Experimente, er findet sich jedoch bereits in den 1930er-Jahren in der Debatte um die Funktionsweise des amerikanischen Föderalismus. Damals wurde davon ausgegangen, dass die amerikanischen Einzelstaaten die Funktion von laboratories of experimen- 
tation übernehmen könnten. Da die 50 Einzelstaaten ein hohes $\mathrm{Ma}$ an Autonomie genießen, wären sie in der Lage, unterschiedliche Politikansätze zu entwickeln und zu testen. In diesem Zusammenhang wird häufig argumentiert, dass sich die subnationale Ebene sehr gut für die Erprobung von Innovationen eignet. Erfolgreiche Experimente können dann nicht nur auf andere Einzelstaaten, sondern auch auf die nationalstaatliche Ebene übertragen werden (Morehouse/Jewell 2004: 177).

Diese Diskussion zeigt erstens, dass Experimente sehr unterschiedliche Formen annehmen können und in vielen Politikfeldern anzutreffen sind. Es geht also keineswegs nur um technologische Innovationen, sondern auch um soziale, politische und ökonomische Experimente (vgl. Castán Broto/Bulkeley 2013b: 1935; Schmidt/Ibert/Kuebart et al. 2016: 6). Zweitens bieten räumlich beschränkte Experimente Vorteile, da sie scheitern können und dürfen, ohne dass dies weitreichende Konsequenzen nach sich ziehen würde. Drittens lässt sich aus dieser Diskussion ableiten, dass selbst erfolgreiche Experimente sich keineswegs automatisch, sondern nur unter bestimmten Voraussetzungen ausbreiten.

Neben der deutschsprachigen Forschung zu Reallaboren konnten sich schon deutlich früher auf internationaler Ebene mehrere ähnlich gelagerte Debatten etablieren, wobei vor allem die von der EU-Kommission geförderten Urban Living Labs zu erwähnen sind. Zudem hat sich die internationale Debatte in den letzten Jahren immer mehr auf die eher grundlagenorientierte Forschung zu (lokalen) Experimenten konzentriert. Der Beitrag zielt daher vor allem darauf $\mathrm{ab}$, die Forschung zu Reallaboren in Deutschland innerhalb der europäischen und internationalen Debatten zu verorten und systematisch mit den Debatten um Urban Living Labs und Experimenten zu vergleichen. Alle untersuchten Ansätze - Reallabore, Urban Living Labs und Experimente beziehen sich zwar nicht ausschließlich auf Städte, diese stehen aber im Zentrum der meisten Projekte. Da zwischen den Ansätzen konzeptionell vielfach Überschneidungen und Gemeinsamkeiten bestehen, soll in diesem Beitrag gezielt herausgearbeitet werden, worin sie sich maßgeblich unterscheiden.

Allen drei Ansätzen ist gemeinsam, dass sie Ausdruck des experimental turn in den Sozial- und Wirtschaftswissenschaften sind und mittel- und langfristig zu urbanen Nachhaltigkeitstransformationen beitragen sollen. Dies setzt voraus, dass die Skalierung der im Rahmen der Projekte entwickelten Innovationen gelingt. Dabei geht es sowohl um die zeitliche als auch um die räumliche Dimension der Skalierung. In zeitlicher Hinsicht besteht die Herausforderung in der institutionellen Verstetigung neuer Ansätze, während es in räumlicher Hinsicht um deren Übertragbarkeit auf andere Kontexte geht.
Zunächst werden die drei Ansätze Reallabore, Urban Living Labs und Experimente vorgestellt, wobei vor allem auf deren Grundannahmen, ihre Entstehungsgeschichte und Anwendungsbereiche sowie die Bedeutung von Lernprozessen eingegangen wird (Kapitel 2). Anschließend werden die Ansätze miteinander verglichen und nach Gemeinsamkeiten und Unterschieden gefragt (Kapitel 3). Überlegungen zum Beitrag von Reallaboren, Urban Living Labs und Experimenten zu urbanen Nachhaltigkeitstransformationen stehen im Mittelpunkt des vierten Kapitels, bevor am Ende einige Schlussfolgerungen zur Verortung und Einbettung der deutschen Diskussion zu Reallaboren in die internationale Debatte gezogen werden (Kapitel 5).

\section{Reallabore, Urban Living Labs und Experimente}

\subsection{Reallabore}

Zwar spielen Reallabore in der deutschsprachigen wissenschaftlichen Debatte bereits seit einiger Zeit eine Rolle, dennoch existiert bislang keine eindeutige Definition (Schäpke/Stelzer/Bergmann et al. 2017: 3). Angesiedelt an der ,Schnittstelle zwischen Wissenschaft, Gesellschaft und Politik“ (Borner/Kraft 2018: 1) handelt es sich bei Reallaboren im Kern um eine Form transdisziplinärer Forschung (Singer-Brodowski/Beecroft/Parodi 2018: 23; Renn 2021: 2) beziehungsweise um eine Forschungsinfrastruktur, in der Realexperimente durchgeführt werden können (Engels/Rogge 2018: 28; Schneidewind/Augenstein/Stelzer et al. 2018: 12), wobei soziales und gesellschaftliches Lernen (Parodi/Beecroft/Albiez et al. 2017: 76; Schäpke/Stelzer/Bergmann et al. 2017: 3-4; Singer-Brodowski/ Beecroft/Parodi 2018: 23), die Koproduktion von Wissen (Borner/Kraft 2018: 8-9; Renn 2018: 1; Schneidewind/ Augenstein/Stelzer et al. 2018: 12) sowie das Ko-Design des Forschungsprozesses (Defila/Di Giulio 2018: 99; Engels/Rogge 2018: 29) von entscheidender Bedeutung sind. Ziel eines Reallabors ist es, auf andere Orte und Kontexte übertragbare Ergebnisse zu generieren, welche das Potenzial haben, einen gesellschaftlichen Transformationsprozess anzustoßen (Renn 2018: 1; Singer-Brodowski/ Beecroft/Parodi 2018: 23-24; Schneidewind/Augenstein/ Stelzer et al. 2018: 12).

Für die erfolgreiche Umsetzung eines Reallaboransatzes sehen viele Forschende die Gleichberechtigung aller beteiligten Interessengruppen als eine zentrale Voraussetzung an (vgl. Engels/Rogge 2018: 29; Renn 2018: 1; Schneidewind/Augenstein/Stelzer et al. 2018: 12; Singer-Brodowski/ Beecroft/Parodi 2018: 26). Darüber hinaus liegt dem Reallaboransatz die Annahme zugrunde, dass die involvierten 
Wissenschaftlerinnen und Wissenschaftler für eine erfolgreiche Projektdurchführung sehr gut mit der Forschungsund Projektumgebung vertraut sind (Renn 2018: 1). Ferner wird davon ausgegangen, dass bei einem typischerweise stark transdisziplinär und akteurzentrierten Reallaborprojekt alle Projektbeteiligten in der Lage sind, sich in die Situation des jeweils anderen zu versetzen (Schäpke/Stelzer/ Bergmann et al. 2016: 48; Engels/Walz 2018: 43-44; Renn 2018: 1; Singer-Brodowski/Beecroft/Parodi 2018: 24-25). Dies erfordert ein gewisses Maß an Offenheit und Reflexivität (Schäpke/Stelzer/Bergmann et al. 2016: 48; SingerBrodowski/Beecroft/Parodi 2018: 24), bedeutet jedoch keineswegs, dass die unterschiedlichen Akteure gezwungen wären, eine einheitliche Sichtweise einzunehmen (Engels/ Walz 2018: 41-43). Vielmehr geht es darum, dass das Verstehen und Akzeptieren unterschiedlicher Perspektiven (innerhalb eines Projekts) den Schlüssel für eine erfolgreiche Zusammenarbeit darstellt (Engels/Walz 2018: 41-43). Inwiefern die aus einem Reallabor gewonnenen Erkenntnisse auf andere Kontexte übertragbar sind, spielte in der deutschsprachigen wissenschaftlichen Diskussion bisher eher eine untergeordnete Rolle.

Bevor sich der Begriff Reallabor in den letzten Jahren etablieren konnte, war bereits seit den späten 1980er-Jahren der aus der Soziologie stammende Begriff des Realexperiments verbreitet (Krohn/Weyer 1989: 349; Groß/HoffmannRiem/Krohn 2005: 12; Schneidewind/Augenstein/Stelzer et al. 2018: 12; Wanner/Stelzer 2019: 5; von Wirth/LevinKeitel 2020: 98). Bei Realexperimenten lag der Fokus auf der Gesellschaft und wie diese (als Ganzes) auf diverse Unsicherheiten der modernen Welt reagiert (Groß/HoffmannRiem/Krohn 2005: 12). Die dahinterliegende Grundannahme war, dass in modernen Gesellschaften im Grunde alle - direkt oder zumindest indirekt - an der Produktion von Wissen beteiligt sind und somit folglich auch die gesamte Gesellschaft in den Forschungsprozess miteinbezogen werden sollte (Krohn/Weyer 1989: 349; Groß/HoffmannRiem/Krohn 2005: 12). Diese Annahme liegt durchaus auch einigen Reallaborprojekten zugrunde. Der Begriff des Reallabors entstammt jedoch nicht ausschließlich der wissenschaftlichen Debatte, sondern wurde auch im Rahmen verschiedener Förderprogramme mit Fokus auf die Bereiche Nachhaltigkeit und Energiewende geprägt (Bauer 2018: 4).

Den Anfang machte das Bundesland Baden-Württemberg, wo 2013 die Expertengruppe „Wissenschaft für Nachhaltigkeit" die Einrichtung von Reallaboren als zentrales Innovationsinstrument der 2014 verabschiedeten Nachhaltigkeitsstrategie empfahl (Ministerium für Umwelt, Klima und Energiewirtschaft Baden-Württemberg 2014: 26; Bauer 2018: 4). Seit 2015 wurden in Baden-Württemberg insgesamt 14 Reallaborprojekte aus den Bereichen klimaverträg- liches Wohnen, Mobilität, demographischer Wandel und Digitalisierung gefördert. Während die erste Förderlinie „Reallabore - BaWü-Labs" noch einen allgemeineren Fokus hatte, legte die zweite Förderlinie „Reallabore Stadt" das Augenmerk explizit auf Städte und städtische Ballungsräume (Wagner/Miller 2018: 5). ${ }^{1}$ Angestoßen durch die Digitalisierungsstrategie des Landes von 2017 wird zudem seit 2019 das Thema Künstliche Intelligenz im Rahmen eines Reallabors gefördert und erforscht (Ministerium für Inneres, Digitalisierung und Migration Baden-Württemberg 2017: 10).

Nahezu zeitgleich wurde auf Bundesebene die „Leitinitiative Zukunftsstadt" eingerichtet, welche zum Ziel hatte, in Zusammenarbeit mit Akteuren aus Wissenschaft, Zivilgesellschaft, Wirtschaft und Kommunen Strategien für eine Nachhaltigkeitstransformation deutscher Städte zu entwickeln (vgl. Bundesministerium für Bildung und Forschung 2015: 3). So wurde etwa die Stadt Dresden im Rahmen des Programms durch zahlreiche Projekte der Stadtverwaltung mit Bürgerinnen und Bürgern in ein Reallabor verwandelt, welches sich aus insgesamt zehn Experimenten zusammensetzte. ${ }^{2}$ Das Beispiel Dresden zeigt exemplarisch, dass Reallaborprojekte oft aus mehreren unterschiedlichen Realexperimenten bestehen, welche in Kooperation von Partnern aus Wissenschaft und Praxis vorbereitet, durchgeführt und analysiert werden (Beecroft/Trenks/Rhodius et al. 2018: 76).

Ab 2019 schrieb der Bund weitere Fördermittel für die Entwicklung von Reallaboren aus. Seitdem werden 20 Reallabore zu den Oberthemen Wasserstofftechnologien, Sektorkopplung, energieoptimierte Quartiere und Energiespeicher als Teil des 7. Energieforschungsprogramms der Bundesregierung vom Wirtschaftsministerium mit insgesamt 100 Millionen Euro gefördert. Ferner wurden weitere 200 Millionen Euro für die Einrichtung zukünftiger Reallabore in traditionellen Energieregionen und Strukturwandelgebieten, wie etwa der Lausitz, zur Verfügung gestellt. $^{3}$

Nicht zuletzt auch wegen der praktischen Relevanz des Themas und der beschriebenen finanziellen Förderung hat sich das Reallabor als Forschungs- und Diskussionsgegenstand auch in der wissenschaftlichen Debatte etabliert. Im

\footnotetext{
1 Vgl. https://www.baden-wuerttemberg.de/de/service/presse/ pressemitteilung/pid/forschung-fuer-nachhaltigkeit-erfolgs modell-reallabor-made-in-bw-1/ (28.06.2021).

2 Vgl. https://www.ioer.de/presse/einzel-2018/?tx_ttnews\%5Btt_ news $\% 5 \mathrm{D}=403$ undcHash $=4 \mathrm{c} 4 \mathrm{~d} 05 \mathrm{e} 2 \mathrm{da} 4 \mathrm{a} 87 \mathrm{deb} 10 \mathrm{f} 2 \mathrm{df77} \mathrm{cacf} 449$ (28.06.2021).

3 https://www.bmwi.de/Redaktion/DE/Pressemitteilungen/2019/ 20190718-altmaier-verkuendet-gewinner-im-ideenwettbewerbreallabore-der-energiewende.html (28.06.2021).
} 
Hinblick auf die disziplinäre Ausrichtung sehen SingerBrodowski, Beecroft und Parodi (2018: 24) Reallabore im Zentrum der Nachhaltigkeitswissenschaften. Ein Blick auf die wissenschaftlichen Profile einschlägiger Wissenschaftlerinnen und Wissenschaftler, die zum Thema Reallabore in den vergangenen Jahren publiziert haben, verdeutlicht den stark interdisziplinären Charakter der bisherigen Forschung. Neben Vertreterinnen und Vertretern der Nachhaltigkeitswissenschaften (vgl. Renn 2018) stechen hierbei vor allem die Disziplinen Soziologie (vgl. Engels/Walz 2018), Wirtschaftswissenschaften (vgl. Schäpke/Bergmann/Stelzer et al. 2018; Schneidewind/Augenstein/Stelzer et al. 2018), Geographie (vgl. Rogga/Zscheischler/Gaasch 2018), Erziehungswissenschaften und Psychologie (Singer-Brodowski/ Beecroft/Parodi 2018; Wanner/Stelzer 2019), aber auch Wissenschaftlerinnen und Wissenschaftler mit einem ingenieurwissenschaftlichen Hintergrund (vgl. Bergmann/Jahn 2017; Beecroft/Trenks/Rhodius et al. 2018; Parodi/Waitz/ Bachinger et al. 2018) heraus. Wie bereits erwähnt, handelt es sich bei Reallaboren um eine überwiegend im deutschsprachigen Raum geführte Debatte. Vor Kurzem wurde jedoch mit der Verwendung des englischen Begriffs RealWorld Labs von einigen deutschsprachigen Wissenschaftlerinnen und Wissenschaftlern der Versuch unternommen, eine Internationalisierung des Diskurses anzustoßen (vgl. Engels/Rogge 2018; Parodi/Waitz/Bachinger et al. 2018; Renn 2018; Rogga/Zscheischler/Gaasch 2018; Schäpke/ Bergmann/Stelzer et al. 2018; Schneidewind/Augenstein/ Stelzer et al. 2018; McCrory/Schäpke/Holmén et al. 2020: $8)$.

Oft wird der transformative und transdisziplinäre Charakter von Reallaboren hervorgehoben, weswegen sich der Einsatz vieler unterschiedlicher Methoden und deren Kombination ausdrücklich empfiehlt (Schäpke/Stelzer/ Bergmann et al. 2016: 48-49; Engels/Walz 2018: 40; Beecroft/Trenks/Rhodius et al. 2018: 76). Generell bieten sich bei Reallaboren verschiedene Methoden der Aktionsforschung an (Schäpke/Stelzer/Bergmann et al. 2017: 49; Scholl/de Kraker/Hoeflehner et al. 2018: 78). Als mögliche Methoden zur Vorbereitung eines Reallabors (insbesondere Identifikation der zu beteiligenden Akteure) empfehlen Borner und Kraft (2018: 11) das Schneeballverfahren, Fokusgruppen sowie Leitfadeninterviews. Für die eigentliche Durchführung bieten sich dann verschiedene Beteiligungsund Diskussionsformate wie World-Cafés, Open Space oder Fishbowl an (Borner/Kraft 2018: 11). Darüber hinaus besteht auch die Möglichkeit und in gewisser Weise auch die Erwartung, dass im Zuge der Durchführung eines Reallabors existierende Methoden weiterentwickelt oder sogar gänzlich neue Methoden eingeführt und erprobt werden (Borner/Kraft 2018: 7; Singer-Brodowski/Beecroft/Parodi 2018: 25; Beecroft/Trenks/Rhodius et al. 2018: 76).
Gerade weil Reallabore vor allem dann zum Einsatz kommen, wenn komplexe und dynamische Problemstellungen vorliegen, für welche noch kein erprobtes Implementierungswissen vorhanden ist, spielen Lernprozesse sowie die Koproduktion von Wissen für die erfolgreiche Umsetzung eines solchen Ansatzes eine zentrale Rolle (Parodi/Beecroft/ Albiez et al. 2017: 74-75; Schäpke/Stelzer/Bergmann et al. 2017: 20; Borner/Kraft 2018: 11; Renn 2018: 1; Schneidewind/Augenstein/Stelzer et al. 2018: 12). Singer-Brodowski, Beecroft und Parodi (2018: 25-26) unterscheiden hinsichtlich der zu beobachtenden Lernprozesse drei Formen: individuelle Kompetenzentwicklung, soziales Lernen sowie inter- und transdisziplinäre Zusammenarbeit. Von großer Bedeutung ist hierbei, dass diese Formen des Lernens in der Regel nicht getrennt voneinander, sondern vielmehr in Kombination stattfinden sollten. Bezogen auf die Koproduktion von Wissen merkt Renn (2018: 1) an, dass erheblich ist, dass alle Projektbeteiligten sich darüber im Klaren sind, welche Art von Wissen sie in den Prozess einbringen können und inwiefern dieses zur Zielerreichung des Projekts beitragen kann. Dies ist aufgrund der Heterogenität der Akteure aus Gesellschaft, Politik und Wissenschaft entscheidend für die Generierung von „sozial robustem Handlungswissen“ (Borner/Kraft 2018: 14). Singer-Brodowski, Beecroft und Parodi (2018: 26) weisen jedoch auch darauf hin, dass bisher kaum Anstrengungen unternommen wurden, die beschriebenen Lernprozesse zu evaluieren.

\subsection{Urban Living Labs}

Bei Urban Living Labs handelt es sich um Orte innerhalb einer Stadt, die geschaffen wurden, um Innovationen in Echtzeit zu testen, mit dem Ziel, mehr über bestimmte Lösungsansätze für gesellschaftliche, wirtschaftliche und ökologische Probleme in städtischen Räumen zu lernen und Nachhaltigkeitstransformationen anzustoßen (Evans/Karvonen 2011: 127; Juujärvi/Pesso 2013: 22; Bulkeley/Coenen/Frantzeskaki et al. 2016: 13; Voytenko/ McCormick/Evans et al. 2016: 45; Wolfram/Frantzeskaki 2016: 1; von Wirth/Fuenfschilling/Frantzeskaki et al. 2019: 229). Der Aspekt des kollektiven Erprobens sowie die Beteiligung unterschiedlicher städtischer Akteure aus Wissenschaft, Politik, Wirtschaft und Zivilgesellschaft stehen bei Urban Living Labs im Vordergrund (Evans/Karvonen 2011: 127; Bulkeley/Castán Broto 2013: 372; Juujärvi/ Pesso 2013: 22; Eskelinen/Garcia Robles/Lindy et al. 2015: 15; Nesti 2018: 310; von Wirth/Fuenfschilling/Frantzeskaki et al. 2019: 231). Der Anspruch von Urban Living Labs geht jedoch oft weit über das bloße Testen von neuen Lösungsansätzen hinaus. So sehen manche Interessengruppen in ihnen auch eine neue und innovative Form städtischer 
Nachhaltigkeits- und Klimapolitik (Evans/Karvonen 2011: 133).

Was alle Urban Living Labs in ihrem Ansatz eint und sie von Reallaboren unterscheidet, ist die explizite Territorialisierung von Innovationen - etwa durch das Ziehen klarer Grenzen für die Ausweisung eines Labors in einem Stadtteil - mit dem Ziel, das Projekt auf eine überschaubare Größe zu begrenzen (Evans/Karvonen 2011: 129-131; Voytenko/McCormick/Evans et al. 2016: 52). Innerhalb eines solchen Urban Living Labs können dann - wie auch in einem klassischen Labor - Experimente überwacht, aufgezeichnet und wiederholt werden. Im Idealfall handelt es sich bei den Ergebnissen der Laborexperimente, also dem unter Laborbedingungen neu erlangten Wissen, um allgemeingültige Erkenntnisse, die folglich auch andernorts Anwendung finden können (Evans/Karvonen 2011: 129-130; Voytenko/ McCormick/Evans et al. 2016: 62).

Zur Beförderung der Verbreitung der im Labor entwickelten Lösungsansätze müssen diese aufbereitet, zusammengefasst und in die Welt außerhalb des Labors getragen werden (Chesbrough 2003: 44). Für die Verbreitung des generierten Wissens spielt die durch das Urban Living Lab erfolgte Demonstration der Wirksamkeit von Lösungsansätzen eine zentrale Rolle (Bulkeley/Coenen/Frantzeskaki et al. 2016: 13; von Wirth/Fuenfschilling/Frantzeskaki et al. 2019: 237). Darüber hinaus besteht die Hoffnung, dass sich durch die Existenz eines oder mehrerer Urban Living Labs auch die Stadt selbst zu einer Stätte der Wissensproduktion und des nachhaltigen und umweltfreundlichen Wirtschaftens entwickelt. Inwiefern die im Labor erzielten Ergebnisse jedoch überhaupt auf andere Stadtteile innerhalb derselben Stadt oder auf andere Städte übertragen werden können, ist weitgehend ungeklärt und wurde bisher wenig erforscht (Evans/ Karvonen 2011: 129). Von Wirth, Fuenfschilling, Frantzeskaki et al. (2019: 250-251) weisen in diesem Zusammenhang darauf hin, dass viele Urban Living Labs überhaupt nicht auf eine Verbreitung über ihre (engen) Grenzen hinaus ausgelegt sind.

Der Begriff Living Lab wurde das erste Mal von Bajgier, Maragah, Saccucci et al. (1991) verwendet. Sie hatten zusammen mit Studierenden der Drexel University in einem Stadtquartier von Philadelphia Lösungsansätze für innerstädtische Strukturprobleme erprobt (Nesti 2018: 313; Abel 2020: 287). Ausgeweitet wurde der Ansatz ab 1995 von William J. Mitchell vom Massachusetts Institute of Technology, der sich mit der Entwicklung und Erprobung von neuen Technologien zum Umgang mit komplexen sozialen Problemen befasste und dessen Ansatz als Modell für unternehmerische Innovationen sowohl in Wissenschaft als auch Praxis auf Interesse stieß (Nesti 2018: 313). Mitchells Verständnis eines Living Labs als nutzerorientierte
Forschungsmethode prägt die Idee des Living Labs bis heute entscheidend (Nesti 2018: 313).

Einzug in die europäische Debatte und Förderlandschaft hielten Living Labs im Jahr 2006. Zunächst wurde auf Betreiben der finnischen Ratspräsidentschaft das European Network of Living Labs (ENOLL) ins Leben gerufen. Wenig später begann die Europäische Kommission im Rahmen ihrer Smart City Strategy mit der Förderung von Living Labs (Nesti 2018: 313).

Während der Begriff Living Lab bereits seit den frühen 1990er-Jahren existierte und später von der Europäischen Union aufgegriffen wurde, handelt es sich bei Urban Living Labs um eine Wortkreation, die im Rahmen entsprechender Förderlinien der Joint Programming Initiative Urban Europe (JPI Urban Europe) entstand. Bei der Programminitiative JPI Urban Europe ging es nicht zuletzt darum, europäischen Städten nach der Finanz- und Wirtschaftskrise ab 2008 die Entwicklung von Visionen städtischer Nachhaltigkeit zu ermöglichen (Voytenko/McCormick/Evans et al. 2016: 47; Nesti 2018: 313). Sicher nicht zuletzt auch wegen der EU-Forschungsförderung hat sich der Begriff Urban Living $L a b$ im wissenschaftlichen Diskurs durchgesetzt (vgl. Evans/Karvonen 2011: 128; Bulkeley/Coenen/Frantzeskaki et al. 2016: 14; Voytenko/McCormick/Evans et al. 2016: 47; Nesti 2018: 313).

In der Folge waren es vor allem Wissenschaftlerinnen und Wissenschaftler aus den Bereichen Politikwissenschaft, Geographie und den Nachhaltigkeitswissenschaften, welche Urban Living Labs erforschten, und besonders aus Schweden, den Niederlanden und Großbritannien (vgl. Evans/Karvonen 2011; Evans 2012; Bulkeley/Coenen/ Frantzeskaki et al. 2016; Voytenko/McCormick/Evans et al. 2016; von Wirth/Fuenfschilling/Frantzeskaki et al. 2019). Inhaltlich dominieren Projekte mit einem Fokus auf Nachhaltigkeit und Dekarbonisierung (Evans/Karvonen 2011: 136; Bulkeley/Coenen/Frantzeskaki et al. 2016: 13; Voytenko/McCormick/Evans et al. 2016: 62; von Wirth/ Fuenfschilling/Frantzeskaki et al. 2019: 239-241). Methodisch stützten sich diese Urban Living Labs vor allem auf die gemeinschaftliche und kontinuierliche Weiterentwicklung von Innovationen durch Brainstorming, Fokusgruppen, Szenarienentwicklung und ethnographische Ansätze, wobei oft auch neuere Informations- und Kommunikationstechnologien (z. B. Apps) zum Einsatz gelangten (Nesti 2015: 273; Nesti 2018: 318).

Ein hoher Stellenwert wird bei Urban Living Labs dem Lernen und kontinuierlichen Lernprozessen beigemessen (Evans/Karvonen 2011: 129-130; Bulkeley/Castán Broto 2013: 367; Juujärvi/Pesso 2013: 23; Voytenko/McCormick/ Evans et al. 2016: 51-52; von Wirth/Fuenfschilling/ Frantzeskaki et al. 2019: 230). Bulkeley, Coenen, Frantzeskaki et al. (2016: 13) merken in diesem Zusammenhang 
an, dass der explizite Fokus auf Wissensgenerierung und Lernen als Kerninstrumente zum Erreichen des verfolgten Projektziels ein Alleinstellungsmerkmal von Urban Living Labs darstellt. Im Gegensatz zu anderen Ansätzen zur Erprobung von Innovationen ist der Lernprozess bei Urban Living Labs stark formalisiert (Bulkeley/Castán Broto 2013: 366-367; Voytenko/McCormick/Evans et al. 2016: 51-52). Die Formalisierung zeigt sich insbesondere durch den rekursiven Charakter der Lernprozesse (Evans/Karvonen 2011: 138). Bei rekursivem Lernen handelt es sich um eine strukturdeterminierte Form des Lernens, die nicht darauf abzielt, etwas gänzlich Neues zu schaffen, sondern sich darauf konzentriert, vorhandenes Wissen stetig weiterzuentwickeln, anzupassen und dadurch zu verbessern (Tenberg 2006: 81). In der Praxis sind die Lernprozesse in Urban Living Labs folglich vor allem durch wiederholtes Ausprobieren und Testen beziehungsweise Learning by Doing gekennzeichnet (Bulkeley/Coenen/Frantzeskaki et al. 2016: 15; Wolfram/Frantzeskaki 2016: 5; Nesti 2018: 321; von Wirth/Fuenfschilling/Frantzeskaki et al. 2019: 243).

Ähnlich wie bei Reallaboren bestehen noch Defizite bezüglich einer systematischen Evaluation der Lernprozesse (Voytenko/McCormick/Evans et al. 2016: 62; von Wirth/ Fuenfschilling/Frantzeskaki et al. 2019: 250-251). Die formalisierten Lernprozesse finden bei Urban Living Labs vor allem zwischen Akteuren aus Forschungseinrichtungen, Politik und Verwaltung, der Privatwirtschaft sowie der Zivilgesellschaft statt, wobei in Forschungseinrichtungen beschäftigte Wissenschaftlerinnen und Wissenschaftler in der Regel die Koordination übernehmen (Juujärvi/Pesso 2013: 23; Eskelinen/Garcia Robles/Lindy et al. 2015: 15; Voytenko/McCormick/Evans et al. 2016: 51-52). Neben dem jeweiligen Projektziel besteht bei Urban Living Labs ein weiteres Ziel oft in der dauerhaften, engen Kooperation zwischen Städten und ihren Universitäten (Evans/Karvonen 2011: 128).

\subsection{Experimente und Experimentieren}

Die Diskussion zu Urban Living Labs hat sich in den letzten Jahren immer mehr der Untersuchung von lokalen Experimenten zugewandt. Die beiden Debatten überschneiden sich vor allem bei der Diskussion zu strategischen Experimenten (Bulkeley/Castán Broto 2013: 366; Marvin/Bulkeley/Mai et al. 2018: 8; von Wirth/Frantzeskaki/ Loorbach 2020: 238-240). Zwar hat sich in dieser Diskussion zu urbanen Experimenten und zum Experimentieren in Städten bislang keine einheitliche Definition durchgesetzt (Hildén/Jordan/Huitema 2017: 2), es lassen sich aber einige zentrale Elemente von Experimenten erkennen. Diese sind (1) innovativ, das heißt, sie umfassen neues Wissen, neue Praktiken, neue Lösungsansätze und neue Akteure;
(2) flexibel, was auch rechtliche Bestimmungen umfasst, da das Handeln nicht von vornherein auf bestimmte Vorgehensweisen beschränkt sein sollte; (3) evidenzbasiert, woraus sich der Vorteil ergibt, dass Innovationen ex ante abgeschätzt werden können; (4) räumlich begrenzt, z. B. auf Nischen, in denen technologische oder soziale Innovationen entwickelt werden; (5) zeitlich begrenzt, da Beginn und Ende der Projekte in der Regel feststehen und (6) reversibel, weil ein Scheitern von Experimenten keine weitreichenden Konsequenzen hat (Caniglia/Schäpke/Lang et al. 2017: 42-44; Hildén/Jordan/Huitema 2017: 2-5; Kivimaa/Hildén/Huitema et al. 2017: 17-18; McFadgen/ Huitema 2018: 162).

Viele Arbeiten gehen von Innovations- und Transitionsansätzen aus und untersuchen die Entstehung von soziotechnischen Innovationen in Nischen und deren Auswirkungen auf den Wandel des Regimes. Hier liegt der Schwerpunkt auf Nachhaltigkeitstransitionen durch technische Innovationen (z. B. Schot/Geels 2008: 546). In einer Literaturstudie entwickeln Kivimaa, Hildén, Huitema et al. (2017: 24) eine Typologie von Experimenten in der Klima-Governance. Demnach dienen Experimente (1) der Schaffung von Nischen, (2) der Schaffung von Märkten, (3) der räumlichen Entwicklung und (4) der Lösung gesellschaftlicher Probleme. Das Autorenteam kommt zu dem Schluss, dass es erforderlich ist, die verschiedenen Typen zu kombinieren. Daneben spielen Governance-Experimente eine wichtige Rolle (z. B. Sabel/Zeitlin 2010: 9-10). Dabei wird angenommen, dass sich Experimente und Experimentieren zu einer eigenständigen Form der Governance urbaner Transformationen entwickelt haben (Fuenfschilling/Frantzeskaki/Coenen 2019: 222; von Wirth/Frantzeskaki/Loorbach 2020: 240). Governance by experiments trägt den unsicheren, komplexen, volatilen und ambivalenten Rahmenbedingungen des Klimawandels und seiner Auswirkungen auf Städte Rechnung.

Neben dem Entwickeln und Erproben neuer Ansätze zielen urbane Experimente zumindest mittel- und langfristig auch auf urbane Transformationen ab (Moloney/ Horne 2015: 2437; Fuenfschilling/Frantzeskaki/Coenen 2019: 220; Hölscher/Frantzeskaki/Loorbach 2019: 791). Während vor allem zu Beginn dieser Debatte nicht hinterfragt wurde, ob und wie (erfolgreiche) Experimente auf andere Kontexte übertragen werden können, rückt die Frage nach den (potenziellen) Beiträgen von Experimenten zu urbanen Transformationen immer mehr in den Mittelpunkt der Diskussion.

Die Bedeutung von Experimenten für politische Reformen wurde in den USA schon seit den 1920er-Jahren thematisiert. So verwies John Dewey, der Philosoph des Experimentalismus, bereits in seinem Werk „The Public and its Problems“" (Dewey 1927) auf den inhärent experi- 
mentellen Charakter der Demokratie (McFadgen/Huitema 2018: 163) und Louis Brandeis bezeichnete in seiner abweichenden Meinung zu einem Urteil des Supreme Court von 1932 die amerikanischen Einzelstaaten als „laboratories of experimentation". ${ }^{4}$ Daraus lässt sich ableiten, dass bereits damals davon ausgegangen wurde, dass es sich bei Experimenten um zeitlich und räumlich begrenzte Projekte handelt.

Die meisten Studien sind eher der Grundlagenforschung als der angewandten Forschung zuzurechnen. Treibend wirkte sicherlich die Förderung durch die EUKommission im Rahmen des COST-Projektes „Innovations in Climate Governance: Sources, Patterns and Effects (INOGOV)“ (2014-2018) sowie die Förderung strategischer Forschung durch die Academy of Finland (Turnheim/ Kivimaa/Berkhout 2018a: xi). ${ }^{5}$ Damit scheint die Diskussion ihren Höhepunkt erreicht zu haben. Seitdem sind weniger Studien $\mathrm{zu}$ urbanen Experimenten erschienen. Darüber hinaus verschiebt sich die Diskussion zusehends auf Fragen zu den Erfolgsbedingungen von Experimenten (z.B. Bai/Roberts/Chen 2010: 322; Peng/Bai 2018: 209-210; Peng/Wei/Bai 2019: 309-310).

Im Gegensatz zu der Diskussion zu Reallaboren sind die Ansätze zu urbanen Experimenten global, europäisch oder zumindest international vergleichend ausgerichtet. So untersucht Hoffmann (2011: 165-171) im Rahmen einer Literaturstudie rund 60 klimapolitische Experimente und zahlreiche transnationale Städtenetzwerke. Castán Broto und Bulkeley (2013a: 92) analysierten weltweit 627 klimapolitische Experimente in 100 Städten. Darüber hinaus wurde eine breite Palette von Fallstudien durchgeführt, wobei es sich häufig um Einzelfallstudien oder eine beschränkte Anzahl von Experimenten handelte. Zwar beziehen sich die Studien nicht nur auf Städte, diese bzw. die lokale Ebene waren jedoch sehr häufig ein Schwerpunkt. Inhaltlich dominieren Experimente im Bereich der Energiepolitik, es wurden aber auch Experimente im Bereich der Mobilität sowie in anderen Politikfeldern betrachtet. Nicht nur staatliche, auch nichtstaatliche Initiativen stehen häufig im Mittelpunkt der Forschung (Bulkeley/Castán Broto 2013: 372; Castán Broto/Bulkeley 2013a: 93; Bulkeley/Castán Broto/Edwards 2015: 10-12; Evans/Karvonen/ Raven 2016: 10-12; Turnheim/Kivimaa/Berkhout 2018b: 19). Kivimaa, Hildén, Huitema et al. (2017: 24) kommen zu dem Schluss, dass die inkonsistente Konzeptionalisie-

\footnotetext{
${ }^{4}$ Vgl. https://supreme.justia.com/cases/federal/us/285/262/ (28.06.2021).

${ }^{5}$ Vgl. auch die Beiträge im special issue der Zeitschrift „Journal of Cleaner Production“ zum Thema „Experimentation for climate change solutions“ im Jahr 2017.
}

rung von Experimenten das kumulative Verständnis über die Fallstudien hinweg erschwert. Aus diesen können daher kaum generalisierbare Ergebnisse abgeleitet werden (Kivimaa/Hildén/Huitema et al. 2017: 26).

Urbane Experimente können als interdisziplinär charakterisiert werden (Turnheim/Kivimaa/Berkhout 2018b: 5-6). Sie umfassen Ansätze wie das aus der Ökologie stammende Konzept des adaptiven Managements ebenso wie den in den Niederlanden entwickelten Transitionsansatz (Smith/Raven 2012: 1025; Seyfang/Hielscher/Hargreaves et al. 2014: 23-24) sowie politikwissenschaftlich und geographisch geprägte Ansätze zur Analyse von Politikfeldern und experimentellen Formen der (Klima-)Governance (Sabel/Zeitlin 2010: 9-10; Hoffmann 2011: 27-32; Bulkeley/Castán Broto 2013: 362-367; Jordan/Huitema 2014: 387-389).

Zwar wird in der Literatur darauf verwiesen, dass Experimente transdisziplinäre Projekte stimulieren können (Hildén/Jordan/Huitema 2017: 2-5), im Gegensatz zu der Debatte um Reallabore und Urban Living Labs ist die Transdisziplinarität dieser Forschungsrichtung jedoch weniger stark ausgeprägt. Daher spielt die Diskussion zur Kooperation zwischen Wissenschaft und Praxis nur eine untergeordnete Rolle. Erwähnt wird die Koproduktion von Wissen und Innovationen, da Nischen Möglichkeiten für Lernprozesse bieten. Peng, Wei und Bai (2019: 306) kommen zu dem Schluss, dass sich der Fokus vom externen Lernen zwischen räumlich getrennten Experimenten zum internen reflexiven Lernen verschiebt, das durch adäquate Formen der Kontextualisierung erleichtert wird. Schließlich geht es im Zusammenhang mit Lernprozessen um die als notwendig erkannte Evaluation der Projekte, die bislang stark vernachlässigt wurde (Kivimaa/Hildén/Huitema et al. 2017: 18; Peng/Bai 2018: 208-209; Peng/Wei/Bai 2019: 306).

\section{Reallabore, Urban Living Labs und Experimente im Vergleich: Gemeinsamkeiten und Unterschiede}

Die Vorstellung, dass es möglich ist, innerhalb einer Stadt ein Labor einzurichten, die allen drei Ansätzen gemeinsam ist, erscheint wenig realistisch, da die ,echte Welt" nicht wie ein Labor funktioniert (Evans/Karvonen 2011: 129). Während ein künstlich geschaffenes und ständig kontrolliertes Labor strikt von der Außenwelt isoliert werden kann und innerhalb eines solchen zudem verschiedene Variablen nach Belieben verändert und dadurch Hypothesen getestet werden können, handelt es sich bei Städten um sich ständig verändernde, mehrdimensionale und offene Systeme (vgl. Evans/Karvonen 2011: 129). Warum also sollte es 
Tabelle 1 Reallabore, Urban Living Labs und lokale Experimente im Vergleich

\begin{tabular}{|c|c|c|c|}
\hline & Reallabore & Urban Living Labs & Lokale Experimente \\
\hline Forschungsschwerpunkte & $\begin{array}{l}\text { Anwendungsorientierte For- } \\
\text { schung }\end{array}$ & $\begin{array}{l}\text { Anwendungsorientierte For- } \\
\text { schung }\end{array}$ & $\begin{array}{l}\text { Anwendungsorientierte Grund- } \\
\text { lagenforschung }\end{array}$ \\
\hline Dominierende Disziplinen & $\begin{array}{l}\text { Nachhaltigkeitswissenschaften, } \\
\text { Wirtschaftswissenschaften, } \\
\text { Geographie, Soziologie, Erzie- } \\
\text { hungswissenschaften, Psycho- } \\
\text { logie, Ingenieurwissenschaften }\end{array}$ & $\begin{array}{l}\text { Politikwissenschaften, Geogra- } \\
\text { phie, Nachhaltigkeitswissen- } \\
\text { schaften }\end{array}$ & $\begin{array}{l}\text { Politikwissenschaften, Geogra- } \\
\text { phie }\end{array}$ \\
\hline Reichweite & Deutschland & $\begin{array}{l}\text { international (insbesondere } \\
\text { EU) }\end{array}$ & global \\
\hline $\begin{array}{l}\text { Dominierende Handlungsfel- } \\
\text { der }\end{array}$ & $\begin{array}{l}\text { Nachhaltigkeit, Energiewende, } \\
\text { Digitalisierung }\end{array}$ & $\begin{array}{l}\text { Nachhaltigkeit, Dekarbonisie- } \\
\text { rung }\end{array}$ & $\begin{array}{l}\text { Energiewende, Dekarbonisie- } \\
\text { rung }\end{array}$ \\
\hline $\begin{array}{l}\text { Territoriale Abgrenzung des } \\
\text { "Labors“ }\end{array}$ & $\begin{array}{l}\text { Stadtquartiere oder Städte; zu- } \\
\text { nehmend auch ganze Regionen } \\
\text { bzw. Teilregionen }\end{array}$ & Stadtquartiere oder Städte & Stadtquartiere oder Städte \\
\hline Treibende Kräfte & $\begin{array}{l}\text { Bundes- und Landesministeri- } \\
\text { en, Wissenschaftlerinnen und } \\
\text { Wissenschaftler }\end{array}$ & $\begin{array}{l}\text { EU-Institutionen, Wissenschaft- } \\
\text { lerinnen und Wissenschaftler }\end{array}$ & $\begin{array}{l}\text { vor allem Wissenschaftlerinnen } \\
\text { und Wissenschaftler }\end{array}$ \\
\hline Beteiligte Akteure & $\begin{array}{l}\text { Wissenschaft, Politik und Ver- } \\
\text { waltung, Wirtschaft, Gesell- } \\
\text { schaft }\end{array}$ & $\begin{array}{l}\text { Wissenschaft, Politik und Ver- } \\
\text { waltung, Wirtschaft, Gesell- } \\
\text { schaft }\end{array}$ & $\begin{array}{l}\text { vor allem Wissenschaft, Politik } \\
\text { und Verwaltung }\end{array}$ \\
\hline
\end{tabular}

überhaupt möglich sein, in einer solchen Umgebung Laborbedingungen herstellen und Ergebnisse generieren $\mathrm{zu}$ können, die bestimmten Gesetzmäßigkeiten folgen oder gar universal anwendbar sind? Tatsächlich kann etwa ein Reallabor, ein Urban Living Lab oder ein lokales Experiment dies nicht leisten und auch sonst ist bisher sehr wenig über ihre konkreten Wirkungen auf Nachhaltigkeitstransformationen bekannt (Bulkeley/Coenen/Frantzeskaki et al. 2016: 16; Voytenko/McCormick/Evans et al. 2016: 49; von Wirth/Fuenfschilling/Frantzeskaki et al. 2019: 250-251; Grunwald/Schäfer/Bergmann 2020: 111-113).

Eine weitere Gemeinsamkeit der drei Ansätze ist darin zu sehen, dass die meisten Projekte bislang nicht systematisch evaluiert wurden (Voytenko/McCormick/Evans et al. 2016: 62; Kivimaa/Hildén/Huitema et al. 2017: 18; SingerBrodowski/Beecroft/Parodi 2018: 26; Turnheim/Kivimaa/ Berkhout 2018b: 21; von Wirth/Fuenfschilling/Frantzeskaki et al. 2019: 250). Dies ist ein wichtiger Grund für das eingeschränkte Wissen um die Bedeutung und die Wirkungen von Reallaboren, Urban Living Labs und Experimenten und erschwert zudem deren Verbreitung. Dass über die Wirkung von Reallaboren und Urban Living Labs bisher so wenig bekannt ist, erstaunt insofern, da nicht zuletzt Lernen und strukturierte Lernprozesse hier eine zentrale Rolle einnehmen und davon ausgegangen werden kann, dass der Schritt von der systematischen Vor- und Nachbereitung von Lernprozessen zwischen den beteiligten Akteuren und der Evaluation eines Projekts nicht allzu groß ist.

Unterschiede zwischen den drei Ansätzen bestehen darin, dass die Debatten zu Reallaboren und Urban Living Labs - im Vergleich zu den Diskussionen zu Experimenten - sehr stark von Forschungsfördereinrichtungen geprägt wurden. Bei Reallaboren waren es vor allem das Ministerium für Umwelt, Klima und Energiewirtschaft Baden-Württemberg, das Bundesministerium für Bildung und Forschung und etwas später das Bundesministerium für Wirtschaft und Energie, die den Begriff im Rahmen verschiedener Förderprogramme einführten und verbreiteten. Der Begriff des Urban Living Labs wurde hingegen stark durch das europäische Programm JPI Urban Europe geprägt. In der Folge hielten beide Forschungsansätze Einzug in die wissenschaftliche Debatte. Im Gegensatz dazu hat die Diskussion um Experimente ihren Ursprung in der Wissenschaft. Nicht zuletzt aufgrund dieser verschiedenen Ausgangsbedingungen handelt es sich bei der Untersuchung von Experimenten um eine stärker grundlagenorientierte Forschungsrichtung, wohingegen Reallabore und Urban Living Labs als deutlich anwendungsorientierter und transdisziplinärer einzuschätzen sind. Zudem ist die Debatte um Experimente eher global ausgerichtet, während Urban Living Labs vor allem auf Europa und Reallabore auf Deutschland begrenzt sind (vgl. Tabelle 1 und Abbildung 1). Diese durchaus substanziellen Unterschiede zwischen den Ansätzen dürfen jedoch nicht verdecken, dass es konzeptionell vielfach Überschneidungen und Gemeinsamkeiten zwischen Reallaboren, Urban Living Labs und Experimenten gibt.

Ein Blick auf aktuelle Publikationen zeigt deutlich, dass sowohl Urban Living Labs als auch Experimente nicht mehr dieselbe Rolle in der wissenschaftlichen Diskussion spielen wie noch vor wenigen Jahren. Da das Bundeswirtschaftsministerium gerade erst mit der Förderung von Reallaboren begonnen hat, ist im Gegensatz dazu schon aufgrund der 


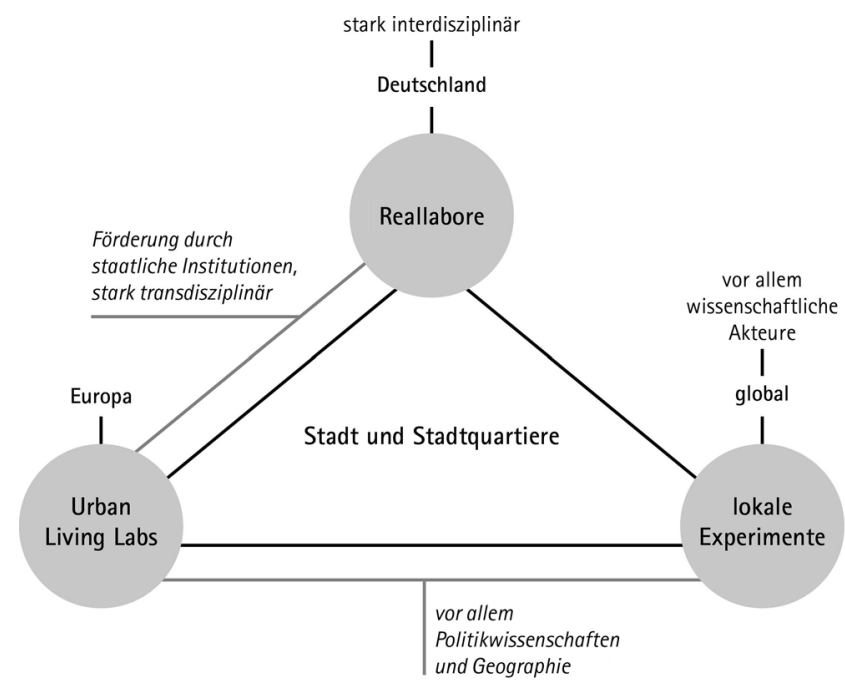

Abbildung 1 Gemeinsamkeiten und Unterschiede zwischen Reallaboren, Urban Living Labs und lokalen Experimenten

praktischen Relevanz dieser Forschungsrichtung damit zu rechnen, dass die in Deutschland geförderten Reallabore in Zukunft noch an Bedeutung gewinnen werden. Vor diesem Hintergrund stellt sich die Frage, inwiefern diese Projekte von den Erfahrungen der deutlich älteren Debatten zu Urban Living Labs und lokalen Experimenten profitieren könnten, was bisher kaum der Fall zu sein scheint (vgl. auch McCrory/Schäpke/Holmén et al. 2020: 8). Generell fällt auf, dass sich die Debatte um Reallabore sehr wenig auf international geführte Diskussionen bezieht. Dies zeigt auch die Tatsache, dass deutsche Wissenschaftlerinnen und Wissenschaftler sowie Forschungseinrichtungen an den internationalen Debatten zu Urban Living Labs und Experimenten bislang kaum beteiligt waren.

\section{Transformation durch Skalierung}

Obgleich sich die drei Ansätze nicht von Anfang an auf Fragen der Skalierung bezogen haben, bestehen weitreichende Erwartungen im Hinblick auf die als notwendig erkannte Transformation in Richtung Nachhaltigkeit. Die bisherigen Ergebnisse abgeschlossener Projekte legen jedoch die Vermutung nahe, dass es sich dabei hauptsächlich um Rhetorik handelt. Gerade für die deutlich ältere Debatte um die Urban Living Labs wurde von Wissenschaftlerinnen und Wissenschaftlern bemängelt, dass Nachhaltigkeitstransformationen weitgehend ausgeblieben sind beziehungsweise der angenommene Zusammenhang zwischen Urban Living Labs und Nachhaltigkeitstransformationen bislang nicht belegt werden konnte (vgl. Voytenko/McCormick/Evans et al. 2016: 49; von Wirth/Fuenfschilling/Frantzeskaki et al. 2019: 250). Vor diesem Hintergrund wird sowohl bei
Reallaboren als auch bei Urban Living Labs von Seiten der Wissenschaft vor zu hohen Erwartungen in die tatsächlichen Wirkungen der durchgeführten Projekte gewarnt (Evans/ Karvonen 2011: 140; Evans/Karvonen/Raven 2016: 4; Dunlop/Radaelli 2018: 266-268; von Wirth/Fuenfschilling/ Frantzeskaki et al. 2019: 250-251; Cairney/Oliver 2020: 237-238).

Transformation setzt die Skalierung von Experimenten voraus, wobei es hier sowohl um die zeitliche als auch die räumliche Dimension der Skalierung geht. Im Hinblick auf die zeitliche Dimension der Skalierung besteht das Problem vor allem darin, dass es sich bei lokalen Experimenten meistens um zeitlich befristete Projekte handelt (z. B. Pilot- und Demonstrationsprojekte). Es stellt sich daher die Frage, wie erfolgreiche Projekte mittel- und langfristig verstetigt werden können. Obgleich die zeitliche Skalierung bei Reallaboren und Urban Living Labs höchst relevant ist, wurde diese Frage bisher kaum thematisiert (Hughes/Yordi/ Besco 2020: 278-280). Die Verstetigung von erfolgreichen Projekten ist in der Regel sehr stark von deren Finanzierung und der dauerhaften Institutionalisierung über das Projektende hinaus abhängig. Dies gilt für urbane Reallabore in verstärktem Maße, da die meisten Kommunen nur über beschränkte Ressourcen für solche neuartigen Formen der Wissensgenerierung und Governance verfügen, die zudem nur schwer mit den traditionell hierarchischen Strukturen deutscher Stadtverwaltungen kompatibel sind. Wenn es gelingt, Reallabore und Experimente in bereits vorhandene Strukturen, Praktiken und Kulturen einzubetten und die Zivilgesellschaft stärker an den Prozessen zu beteiligen, steigen die Chancen ihrer Verstetigung.

Die räumliche Dimension der Skalierung bzw. die räumliche Begrenzung von Reallaboren, Urban Living Labs und Experimenten hat zur Folge, dass auch die Ergebnisse erfolgreicher Projekte häufig nicht direkt auf andere Kontexte übertragen werden können. Es stellt sich also grundsätzlich die Frage, inwiefern neu gewonnenes Wissen auch andernorts angewandt werden kann. Viele Studien erwähnen zwar die Notwendigkeit der räumlichen Skalierung von Innovationen, eine systematische Analyse dieser Fragen liefern viele Studien jedoch noch nicht (Hildén/Jordan/Huitema 2017: 4; van Winden/van den Buuse 2017: 51; Peng/Bai 2018: 209-210; van Doren/Driessen/Runhaar et al. 2018: 175; Peng/Wei/Bai 2019: 309-310; Hughes/Yordi/Besco 2020: 278-280; Lam/Martín-López/Wiek et al. 2020: 2-3). Soll der Transfer gelingen, darf er nicht als einmalige Handlung verstanden werden. Vielmehr ist die Fähigkeit zu kontinuierlichem und iterativem Handeln unter sich dynamisch verändernden Kontextbedingungen erforderlich (vgl. Hughes/ Yordi/Besco 2020: 289).

Außerdem müssen Überlegungen zur räumlichen Skalierung stärker mit institutionellen Fragen verknüpft werden, 
da zwischen Reallaboren, Urban Living Labs und Experimenten auf der einen Seite und Institutionen auf der anderen Seite interdependente Beziehungen bestehen. Institutionen beeinflussen Experimente, und umgekehrt können Experimente zu institutionellem Wandel beitragen (einschließlich notwendiger De-Institutionalisierungen) und so politische Entscheidungen beeinflussen (z. B. McFadgen/ Huitema 2018: 161; Fuenfschilling/Frantzeskaki/Coenen 2019: 219).

Insgesamt bedeutet dies, dass bei allen drei Ansätzen Defizite bei der systematischen Berücksichtigung der zeitlichen und räumlichen Skalierung der Projekte bestehen, und dies sowohl in konzeptioneller als auch in praktischer Hinsicht. Daher kann ein unmittelbarer Kausalzusammenhang zwischen Projekten und Nachhaltigkeitstransformationen bislang nicht nachgewiesen werden. Mittel- und langfristige Veränderungen in Richtung Nachhaltigkeit setzen die Skalierung von Projekten voraus, die wiederum sehr stark davon abhängt, ob es gelingt, diese sowohl zeitlich als auch räumlich in die vorhandenen institutionellen Arrangements einzubetten. Da sich die internationale wie auch die deutsche Diskussion zu lokalen Experimenten immer mehr auf die Frage der Skalierung konzentriert, könnten sich die drei hier diskutierten Forschungsansätze Reallabor, Urban Living Labs und Experimente gegenseitig befruchten.

\section{Schlussfolgerungen}

Dieser Beitrag hat die deutschsprachige Forschung zu Reallaboren innerhalb der europäischen und internationalen Debatten verortet und systematisch mit den konzeptionell ähnlichen Debatten zu Urban Living Labs und Experimenten verglichen.

Zwar existieren bei Reallaboren sehr hohe Erwartungen im Hinblick auf das Potenzial solcher Projekte für die mittel- und langfristige Transformation in Richtung Nachhaltigkeit, ob sie tatsächlich solche Wirkungen entfalten können, ist jedoch eine weitgehend offene Frage. Der Vergleich der deutschen Diskussion zu Reallaboren mit den internationalen Debatten zu Urban Living Labs und zu Experimenten zeigt zunächst einmal, dass bei allen drei Ansätzen deutliche Defizite bezüglich Evaluation der Projekte bestehen.

Da Nachhaltigkeitstransformationen, die zeitliche und räumliche Skalierungen von Experimenten voraussetzen, sollten die damit verbundenen Forschungsfragen sowohl in konzeptioneller als auch in praktischer Hinsicht bereits in das Forschungsdesign der Projekte integriert werden. Hier wäre ein besserer Anschluss der deutschen Diskussion an die internationale Debatte um Skalierung als Voraussetzung für urbane Nachhaltigkeitstransformationen wünschenswert.
Es fällt auf, dass in Deutschland zwar sehr viele anwendungsbezogene Projekte gefördert werden, deren Erfolg aber auch von Fragen abhängt, die eher der Grundlagenforschung zuzuordnen sind. Diese wird in diesem Bereich in Deutschland bislang jedoch kaum gefördert und betrieben. Daher dominieren in der internationalen Debatte Wissenschaftlerinnen und Wissenschaftler aus Forschungseinrichtungen aus Großbritannien, den Niederlanden und den nordischen Ländern, während in Deutschland beschäftigte Wissenschaftlerinnen und Wissenschaftler bislang kaum präsent sind.

Allerdings könnten deutsche Projekte zu Reallaboren von einer Internationalisierung durchaus profitieren. Hier sind nicht nur die Wissenschaftlerinnen und Wissenschaftler selbst, sondern auch die Fördereinrichtungen gefordert, gemeinsam die Voraussetzungen dafür zu schaffen, dass die eher grundlagenorientierte Forschung zu Experimenten mit der eher anwendungsorientierten Forschung zu Reallaboren verbunden werden kann, da gerade die Kombination beider Ansätze am ehesten Erfolg verspricht.

Schließlich geht es um eine dauerhafte Veränderung der Beziehungen zwischen Forschungseinrichtungen und Städten. Hier scheinen institutionalisierte Formen der Zusammenarbeit von besonderer Bedeutung zu sein. Erstens sind Städte - vor allem in Bereichen wie der Klimapolitik - immer mehr von wissenschaftlicher Expertise abhängig, wobei hier der externen Finanzierung ein hoher Stellenwert zukommt, da die meisten deutschen Kommunen nicht über die notwendigen personellen und finanziellen Ressourcen verfügen. Zweitens setzt transdisziplinäre Forschung voraus, dass Wissenschaftlerinnen und Wissenschaftler lernen, mit Praxispartnern dauerhaft zu kooperieren, was zusätzliche Qualifikationen erforderlich macht, aber auch neue Karrierewege eröffnet. Drittens besteht die Herausforderung vor allem darin, institutionalisierte Formen der Kooperation zwischen Wissenschaft und Praxis zu entwickeln und langfristig zu etablieren. Dies reicht von Kooperationsverträgen zwischen Stadtverwaltung und Universität bis hin zu eigenständigen Forschungsprogrammen wie dem Turku Urban Research Programme, das von der Stadt Turku (Finnland) gemeinsam mit den beiden großen vor Ort ansässigen Universitäten getragen wird. Auch hier könnte die deutsche Diskussion zu Reallaboren von einer Internationalisierung der Debatte profitieren.

\section{Literatur}

Abel, P. (2020): Living Lab: Forschung und Praxis am Universitätscampus. In: HMD Praxis der Wirtschaftsinformatik 57, 2, 285-295. https://doi.org/10.1365/s40702020-00593-z 
Bai, X.; Roberts, B.; Chen, J. (2010): Urban sustainability experiments in Asia: patterns and pathways. In: Environmental Science und Policy 13, 4, 312-325. https://doi. org/10.1016/j.envsci.2010.03.011

Bajgier, S. M.; Maragah, H. D.; Saccucci, M. S.; Verzilli, A.; Prybutok, V. R. (1991): Introducing students to community operations research by using a city neighborhood as a living laboratory. In: Operations Research 39, 5, 701-709. https://doi.org/10.1287/opre.39.5.701

Bauer, T. (2018): Research on Real-World Laboratories in Baden-Württemberg. In: GAIA - Ecological Perspectives for Science and Society 27, S1, 4. https://doi.org/ 10.14512/gaia.27.S1.2

Beecroft, R.; Trenks, H.; Rhodius, R.; Benighaus, C.; Parodi, O. (2018): Reallabore als Rahmen transformativer und transdisziplinärer Forschung: Ziele und Designprinzipien. In: Di Giulio, A.; Defila, R. (Hrsg.): Transdisziplinär und transformativ forschen. Wiesbaden, 75-100. https://doi.org/10.1007/978-3-658-21530-9_4

Bergmann, M.; Jahn, T. (2017): Frameworks for transdisciplinary research: Framework \#2. In: GAIA - Ecological Perspectives for Science and Society 26, 4, 304. https:// doi.org/10.14512/gaia.26.4.3

Borner, J.; Kraft, A. H. (2018): Konzeptpapier zum Reallabor-Ansatz. http://komob.de/wp-content/uploads/2018/ 08/ENavi_Reallabore_Borner-Kraft.pdf

Bulkeley, H.; Castán Broto, V. (2013): Government by experiment? Global cities and the governing of climate change. In: Transactions of the Institute of British Geographers 38, 3, 361-375. https://doi.org/10.1111/j.14755661.2012.00535.x

Bulkeley, H.; Castán Broto, V.; Edwards, G. A. S. (2015): An Urban Politics of Climate Change. Experimentation and the Governing of Socio-technical Transitions. London.

Bulkeley, H.; Coenen, L.; Frantzeskaki, N.; Hartmann, C.; Kronsell, A.; Mai, L.; Marvin, S.; McCormick, K.; van Steenbergen, F.; Voytenko Palgan, Y. (2016): Urban living labs: governing urban sustainability transitions. In: Current Opinion in Environmental Sustainability 22, 13-17. https://doi.org/10.1016/j.cosust.2017.02.003

Bundesministerium für Bildung und Forschung (2015): Zukunftsstadt. Strategische Forschungs- und Innovationsagenda. Berlin.

Cairney, P.; Oliver, K. (2020): How should academics engage in policymaking to achieve impact? In: Political Studies Review 18, 2, 228-244. https://doi.org/10.1177/ 1478929918807714

Caniglia, G.; Schäpke, N.; Lang, D. J.; Abson, D. J.; Luederitz, C.; Wiek, A.; Laubichler, M. D.; Gralla, F.; von Wehrden, H. (2017): Experiments and evidence in sustainability science: A typology. In: Journal of Cleaner
Production 169, 39-47. https://doi.org/10.1016/j.jclepro. 2017.05.164

Castán Broto, V.; Bulkeley, H. (2013a): A survey of urban climate change experiments in 100 cities. In: Global Environmental Change 23, 1, 92-102. https://doi.org/10. 1016/j.gloenvcha.2012.07.005

Castán Broto, V.; Bulkeley, H. (2013b): Maintaining climate change experiments: urban political ecology and the everyday reconfiguration of urban infrastructure. In: International Journal of Urban and Regional Research 37, 6, 1934-1948. https://doi.org/10.1111/1468-2427.12050

Chesbrough, H. W. (2003): Open Innovation. The New Imperative for Creating and Profiting from Technology. Boston.

Defila, R.; Di Giulio, A. (2018): What Is It Good For? Reflecting and Systematizing Accompanying Research to Research Programs. In: GAIA - Ecological Perspectives for Science and Society 27, S1, 97-104. https://doi.org/ 10.14512/gaia.27.S1.17

Dewey, J. (1927): The Public and its Problems. An Essay in Political Inquiry. University Park.

Dunlop, C. A.; Radaelli, C. M. (2018): The lessons of policy learning: types, triggers, hindrances and pathologies. In: Policy and Politics 46, 2, 255-272. https://doi.org/10. 1332/030557318X15230059735521

Engels, A.; Walz, K. (2018): Dealing with multi-perspectivity in Real-World Laboratories: experiences from the transdisciplinary research project 'Urban Transformation Laboratories'. In: GAIA - Ecological Perspectives for Science and Society 27, S1, 39-45. https://doi.org/10. 14512/gaia.27.S1.10

Engels, F.; Rogge, J.-C. (2018): Tensions and trade-offs in Real-World Laboratories: the participants' perspective. In: GAIA - Ecological Perspectives for Science and Society 27, S1, 28-31. https://doi.org/10.14512/gaia.27.S1. 8

Eskelinen, J.; Garcia Robles, A.; Lindy, I.; Marsh, J. B.; Muente-Kunigami, A. (2015): Citizen-driven Innovation. A Guidebook for City Mayors and Public Administrators. Washington D.C.

Evans, J. (2012): Environmental Governance. London.

Evans, J.; Karvonen, A. (2011): Living laboratories for sustainability: Exploring the politics and epistemology of urban adaptation. In: Bulkeley, H.; Castán Broto, V.; Hodson, M.; Marvin, S. (Hrsg.): Cities and Low Carbon Transitions. London, 126-141. = Routledge Studies in Human Geography 35.

Evans, J.; Karvonen, A.; Raven, R. (2016): The experimental city. New modes and prospects of urban transformation. In: Evans, J.; Karvonen, A.; Raven, R. (Hrsg.): The Experimental City. London, 1-12.

Fuenfschilling, L.; Frantzeskaki, N.; Coenen, L. (2019): Ur- 
ban experimentation and sustainability transitions. In: European Planning Studies 27, 2, 219-228. https://doi. org/10.1080/09654313.2018.1532977

Groß, M.; Hoffmann-Riem, H.; Krohn, W. (2005): Realexperimente. Ökologische Gestaltungsprozesse in der Wissensgesellschaft. Bielefeld. https://doi.org/10.14361/ 9783839403044

Grunwald, A.; Schäfer, M.; Bergmann, M. (2020): Neue Formate transdisziplinärer Forschung. Ausdifferenzierte Brücken zwischen Wissenschaft und Praxis. In: GAIA Ecological Perspectives for Science and Society 29, 2, 106-114. https://doi.org/10.14512/gaia.29.2.8

Hildén, M.; Jordan, A.; Huitema, D. (2017): Special issue on experimentation for climate change solutions editorial: The search for climate change and sustainability solutions - the promise and the pitfalls of experimentation. In: Journal of Cleaner Production 169, 1-7. https://doi. org/10.1016/j.jclepro.2017.09.019

Hölscher, K.; Frantzeskaki, N.; Loorbach, D. (2019): Steering transformations under climate change: capacities for transformative climate governance and the case of Rotterdam, the Netherlands. In: Regional Environmental Change 19, 3, 791-805. https://doi.org/10.1007/s10113018-1329-3

Hoffmann, M. J. (2011): Climate Governance at the Crossroads. Experimenting with a Global Response after Kyoto. Oxford.

Hughes, S.; Yordi, S.; Besco, L. (2020): The role of pilot projects in urban climate change policy innovation. In: Policy Studies Journal 48, 2, 271-297. https://doi.org/ 10.1111/psj.12288

Jordan, A.; Huitema, D. (2014): Policy innovation in a changing climate: sources, patterns and effects. In: Global Environmental Change 29, 387-394. https://doi.org/10. 1016/j.gloenvcha.2014.09.005

Juujärvi, S.; Pesso, K. (2013): Actor roles in an urban living lab: What can we learn from Suurpelto, Finland? In: Technology Innovation Management Review 3, 11, 22-27. https://doi.org/10.22215/timreview/742

Kivimaa, P.; Hildén, M.; Huitema, D.; Jordan, A.; Newig, J. (2017): Experiments in climate governance: A systematic review of research on energy and built environment transitions. In: Journal of Cleaner Production 169, 17-29. https://doi.org/10.1016/j.jclepro.2017.01.027

Krohn, W.; Weyer, J. (1989): Gesellschaft als Labor: Die Erzeugung sozialer Risiken durch experimentelle Forschung. In: Soziale Welt 40, 3, 349-373.

Lam, D. P.; Martín-López, B.; Wiek, A.; Bennett, E. M.; Frantzeskaki, N.; Horcea-Milcu, A.; Lang, D. J. (2020): Scaling the impact of sustainability initiatives: a typology of amplification processes. In: Urban Transformations, 2, 3. https://doi.org/10.1186/s42854-020-00007-9
Marvin, S.; Bulkeley, H.; Mai, L.; McCormick, K.; Voytenko Palgan, Y. (2018): Introduction. In: Marvin, S.; Bulkeley, H.; Mai, L.; McCormick, K.; Voytenko Palgan, Y. (Hrsg.): Urban Living Labs. Experimentation with City Futures. London, 1-17.

McCrory, G.; Schäpke, N.; Holmén, J.; Holmberg, J. (2020): Sustainability-oriented labs in real-world contexts: An exploratory review. In: Journal of Cleaner Production 277, 123202, 1-18. https://doi.org/10.1016/ j.jclepro.2020.123202

McFadgen, B.; Huitema, D. (2018): Experimentation at the interface of science and policy: A multi-case analysis of how policy experiments influence political decisionmakers. In: Policy Sciences 51, 2, 161-187. https://doi. org/10.1007/s11077-017-9276-2

Ministerium für Inneres, Digitalisierung und Migration Baden-Württemberg (2017): digital@bw. Digitalisierungsstrategie der Landesregierung Baden-Württemberg. Stuttgart.

Ministerium für Umwelt, Klima und Energiewirtschaft Baden-Württemberg (2014): Nachhaltigkeitsstrategie Baden-Württemberg. Stuttgart.

Moloney, S.; Horne, R. (2015): Low carbon urban transitioning: from local experimentation to urban transformation? In: Sustainability 7, 3, 2437-2453. https://doi.org/ $10.3390 /$ su 7032437

Morehouse, S. M.; Jewell, M. E. (2004): States as laboratories: a reprise. In: Annual Review of Political Science 7, 177-203. https://doi.org/10.1146/annurev.polisci.7. 012003.104913

Nesti, G. (2015): Living labs: A new tool for co-production? In: Bisello, A.; Vettorato, D.; Stephens, R.; Elisei, P. (Hrsg.): Smart and Sustainable Planning for Cities and Regions. Cham, 267-281. https://doi.org/10.1007/9783-319-44899-2_16

Nesti, G. (2018): Co-production for innovation: The urban living lab experience. In: Policy and Society 37, 3, 310-325. https://doi.org/10.1080/14494035.2017. 1374692

Parodi, O.; Beecroft, R.; Albiez, M.; Quint, A.; Seebacher, A.; Tamm, K.; Waitz, C. (2017): The ABC of real-world lab methodology: from 'action research' to 'participation' and beyond. In: Trialog 3-4, 74-82.

Parodi, O.; Waitz, C.; Bachinger, M.; Kuhn, R.; MeyerSoylu, S.; Alcántara, S.; Rhodius, R. (2018): Insights into and recommendations from three Real-World Laboratories: An experience-based comparison. In: GAIA Ecological Perspectives for Science and Society 27, S1, 52-59. https://doi.org/10.14512/gaia.27.S1.12

Peng, Y.; Bai, X. (2018): Experimenting towards a low-carbon city: Policy evolution and nested structure of inno- 
vation. In: Journal of Cleaner Production 174, 201-212. https://doi.org/10.1016/j.jclepro.2017.10.116

Peng, Y.; Wei, Y.; Bai, X. (2019): Scaling urban sustainability experiments: contextualization as an innovation. In: Journal of Cleaner Production 227, 302-312. https:// doi.org/10.1016/j.jclepro.2019.04.061

Renn, O. (2018): Real-World Laboratories - the road to transdisciplinary research? In: GAIA - Ecological Perspectives for Science and Society 27, S1, 1. https://doi. org/10.14512/gaia.27.S1.1

Renn, O. (2021): Transdisciplinarity: Synthesis towards a modular approach. In: Futures 130, 102744, 1-18. https://doi.org/10.1016/j.futures.2021.102744

Rogga, S.; Zscheischler, J.; Gaasch, N. (2018): How much of the Real-World Laboratory is hidden in current transdisciplinary research? In: GAIA - Ecological Perspectives for Science and Society 27, S1, 18-22. https://doi. org/10.14512/gaia.27.S1.6

Sabel, C. F.; Zeitlin, J. (2010): Learning from difference: The new architecture of experimentalist governance in the EU. In: Sabel, C.F.; Zeitlin, J. (Hrsg.): Experimentalist Governance in the European Union. Towards a New Architecture. Oxford, 1-28.

Schäpke, N.; Bergmann, M.; Stelzer, F.; Lang, D. J. (2018): Labs in the real world: Advancing transdisciplinary research and sustainability transformation: Mapping the field and emerging lines of inquiry. In: GAIA - Ecological Perspectives for Science and Society 27, S1, 8-11. https://doi.org/10.14512/gaia.27.S1.4

Schäpke, N.; Stelzer, F.; Bergmann, M.; Lang, D. (2016): Tentative Theses on Transformative Research in RealWorld Laboratories. In: TATuP - Zeitschrift für Technikfolgenabschätzung in Theorie und Praxis 25, 3, 45-51. https://doi.org/10.14512/tatup.25.3.45

Schäpke, N.; Stelzer, F.; Bergmann, M.; Singer-Brodowski, M.; Wanner, M.; Caniglia, G.; Lang, D. J. (2017): Reallabore im Kontext transformativer Forschung. Ansatzpunkte zur Konzeption und Einbettung in den internationalen Forschungsstand. Lüneburg. = IETSR Discussion Papers in Transdisciplinary Sustainability Research 1/2017.

Schmidt, S.; Ibert, O.; Kuebart, A.; Kühn, J. (2016): Open Creative Labs in Deutschland: Typologisierung, Verbreitung und Entwicklungsbedingungen. Erkner.

Schneidewind, U.; Augenstein, K.; Stelzer, F.; Wanner, M. (2018): Structure Matters: Real-World Laboratories as a New Type of Large-Scale Research Infrastructure: A Framework Inspired by Giddens' Structuration Theory. In: GAIA - Ecological Perspectives for Science and Society 27, S1, 12-17. https://doi.org/10.14512/gaia.27.S1. 5

Scholl, C.; de Kraker, J.; Hoeflehner, T.; Wlasak, P.; Drage, T.; Eriksen, M. A: (2018): Transitioning urban experi- ments: Reflections on doing action research with urban labs. In: GAIA - Ecological Perspectives for Science and Society 27, S1, 78-84. https://doi.org/10.14512/gaia.27. S1.15

Schot, J.; Geels, F. W. (2008): Strategic niche management and sustainable innovation journeys: theory, findings, research agenda, and policy. In: Technology Analysis und Strategic Management 20, 5, 537-554. https://doi.org/ 10.1080/09537320802292651

Seyfang, G.; Hielscher, S.; Hargreaves, T.; Martiskainen, M.; Smith, A. (2014): A grassroots sustainable energy niche? Reflections on community energy in the UK. In: Environmental Innovation and Societal Transitions 13, 21-44. https://doi.org/10.1016/j.eist.2014.04.004

Singer-Brodowski, M.; Beecroft, R.; Parodi, O. (2018): Learning in Real-World Laboratories: a systematic impulse for discussion. In: GAIA - Ecological Perspectives for Science and Society 27, S1, 23-27. https://doi.org/ 10.14512/gaia.27.S1.7

Smith, A.; Raven, R. (2012): What is protective space? Reconsidering niches in transitions to sustainability. In: Research Policy 41, 6, 1025-1036. https://doi.org/10.1016/ j.respol.2011.12.012

Tenberg, R. (2006): Didaktik lernfeldstrukturierten Unterrichts. Theorie und Praxis beruflichen Lernens und Lehrens. Bad Heilbrunn.

Turnheim, B.; Kivimaa, P.; Berkhout, F. (2018a): Preface. In: Turnheim, B.; Kivimaa, P.; Berkhout, F. (Hrsg.): Innovating Climate Governance. Moving beyond Experiments. Cambridge, xi-xii. https://doi.org/10.1017/ 9781108277679.001

Turnheim, B.; Kivimaa, P.; Berkhout, F. (2018b): Beyond experiments: innovation in climate governance. In: Turnheim, B.; Kivimaa, P.; Berkhout, F. (Hrsg.): Innovating Climate Governance. Moving beyond Experiments. Cambridge, 1-26. https://doi.org/10.1017/9781108277679. 002

van Doren, D.; Driessen, P. P.; Runhaar, H.; Giezen, M. (2018): Scaling-up low-carbon urban initiatives: towards a better understanding. In: Urban Studies 55, 1, 175-194. https://doi.org/10.1177/0042098016640456

van Winden, W.; van den Buuse, D. (2017): Smart city pilot projects: exploring the dimensions and conditions of scaling up. In: Journal of Urban Technology 24, 4, 51-72. https://doi.org/10.1080/10630732.2017.1348884

von Wirth, T.; Frantzeskaki, N.; Loorbach, D. (2020): Urban living labs as inter-boundary spaces for sustainability transitions? In: de Roo, G.; Yamu, C.; Zuidema, C. (Hrsg.): Handbook on Planning and Complexity. Cheltenham, 237-257.

von Wirth, T.; Fuenfschilling, L.; Frantzeskaki, N.; Coenen, L. (2019): Impacts of urban living labs on sustainabil- 
ity transitions: mechanisms and strategies for systemic change through experimentation. In: European Planning Studies 27, 2, 229-257. https://doi.org/10.1080/ 09654313.2018 .1504895

von Wirth, T.; Levin-Keitel, M. (2020): Lokale Nachhaltigkeitsexperimente als raumwirksame Interventionen: Theoretische Grundlagen und Handlungskonzepte. In: GAIA - Ecological Perspectives for Science and Society 29, 2, 98-105. https://doi.org/10.14512/gaia.29.2.7

Voytenko, Y.; McCormick, K.; Evans, J.; Schliwa, G. (2016): Urban living labs for sustainability and low carbon cities in Europe: towards a research agenda. In: Journal of Cleaner Production 123, 45-54. https://doi. org/10.1016/j.jclepro.2015.08.053
Wagner, F.; Miller, E. (2018): The background and history of Real-World Laboratory funding in Baden-Württemberg. In: GAIA - Ecological Perspectives for Science and Society 27, S1, 5. https://doi.org/10.14512/gaia.27. $\mathrm{S} 1.2$

Wanner, M.; Stelzer, F. (2019): Reallabore - Perspektiven für ein Forschungsformat im Aufwind. Wuppertal. = in brief $07 / 2019$.

Wolfram, M.; Frantzeskaki, N. (2016): Cities and systemic change for sustainability: prevailing epistemologies and an emerging research agenda. In: Sustainability 8, 2, 144. 